\title{
Sensitivity and specificity of self-reported psychiatric diagnoses amongst patients treated for opioid use disorder
}

Kelly Leung ${ }^{1 \dagger}$, Emily Xu $^{1+}$, Tea Rosic ${ }^{2,3}$, Andrew Worster ${ }^{1,3}$, Lehana Thabane ${ }^{3,4,5}$ and Zainab Samaan²,3*

\begin{abstract}
Background: Patients with opioid use disorder (OUD) frequently present with comorbid psychiatric illnesses which have significant implications for their treatment outcomes. Notably, these are often identified by self-report. Our study examined the sensitivity and specificity of self-reported psychiatric diagnoses against a structured diagnostic interview in a cohort of patients receiving outpatient pharmacological treatment for OUD.

Methods: Using cross-sectional data from adults receiving outpatient opioid agonist treatment for OUD in clinics across Ontario, Canada, we compared participants' self-reported psychiatric diagnoses with those identified by the Mini Neuropsychiatric Interview (MINI) Version 6.0 administered at the time of study entry. Sensitivity and specificity were calculated for self-report of psychiatric diagnoses.

Results: Amongst a sample of 683 participants, 24\% $(n=162)$ reported having a comorbid psychiatric disorder. Only 104 of these 162 individuals (64\%) reporting a comorbidity met criteria for a psychiatric disorder as per the MINI; meanwhile, 304 (75\%) participants who self-reported no psychiatric comorbidity were in fact identified to meet MINI criteria for a psychiatric disorder. The sensitivity and specificity for any self-reported psychiatric diagnoses were $25.5 \%$ (95\% Cl 21.3, 30.0) and 78.9\% (95\% Cl 73.6, 83.6), respectively.

Conclusions: Our findings raise questions about the utility of self-reported psychiatric comorbidity in patients with OUD, particularly in the context of low sensitivity of self-reported diagnoses. Several factors may contribute to this including remittance and relapse of some psychiatric illnesses, underdiagnosis, and the challenge of differentiating psychiatric and substance-induced disorders. These findings highlight that other methods should be considered in order to identify comorbid psychiatric disorders in patients with OUD.
\end{abstract}

Keywords: Opioid use disorder, Psychiatric diagnoses, Self-reported diagnoses

\footnotetext{
* Correspondence: samaanz@mcmaster.ca

${ }^{\dagger}$ Kelly Leung and Emily Xu contributed equally to this work.

${ }^{2}$ Department of Psychiatry and Behavioral Neurosciences, McMaster

University, Hamilton, Ontario, Canada

${ }^{3}$ Department of Health Research Methods, Evidence, and Impact, McMaster

University, Hamilton, Ontario, Canada

Full list of author information is available at the end of the article
}

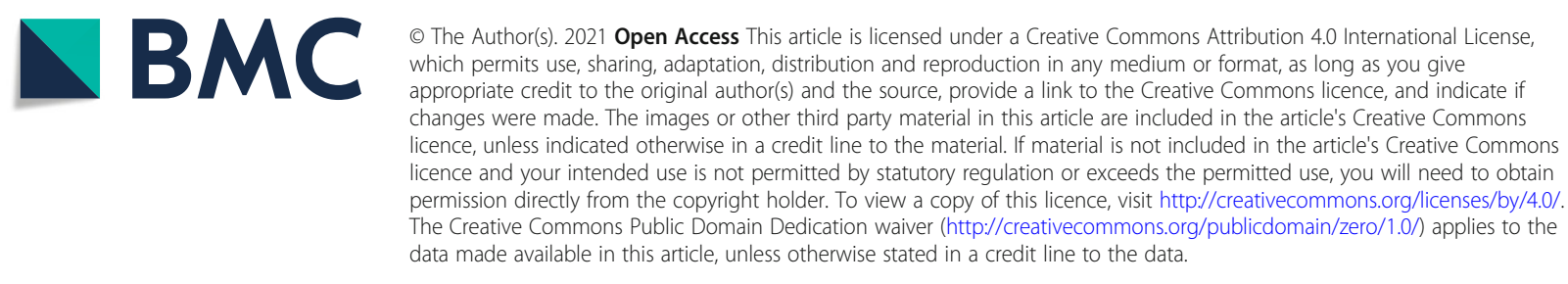




\section{Background}

The opioid crisis continues to be a major public health problem worldwide [1-4]. In 2017, it was estimated that 40.5 million people globally were dependent on opioids, although trends varied between countries [1]. The burden of opioid use and dependence is significantly more prevalent in North America compared to other parts of the world [1]. In Canada, overdose deaths and hospitalizations related to opioid use disorder (OUD) have remained high despite increasing awareness and intervention $[5,6]$. In the United States, opioids are involved in the majority of deaths related to drug overdose, which rose to an all-time high in 2019 with 49,860 overdose deaths involving opioids [7-9]. The impacts of the COVID-19 pandemic have additionally exacerbated preexisting issues relating to access to appropriate treatment and interventions, ensuring that the opioid crisis will remain an ongoing concern for patients and families, healthcare workers, and policymakers alike [9].

Patients with substance use disorders (SUD) experience comorbid psychiatric disorders, such as depression and anxiety, at rates higher than the general population [10]. Canadian data suggest that the 12-month prevalence of psychiatric comorbidity is 5 to 6 times higher among individuals with substance dependence compared to the general population (OR 5.29, 95\% CI 3.90, 7.17) [10]. Additionally, patients with psychiatric disorders report higher rates of substance use problems and are approximately twice as likely to have SUDs $[10,11]$. This comorbidity is exacerbated in patients with OUD who appear to have even higher lifetime prevalence of psychiatric disorders compared to those with other SUDs and the general population; lifetime rates are generally greater than $40 \%$, although there is considerable variability in reported rates across studies [12]. We have previously found that among patients receiving methadone maintenance treatment for OUD, approximately $80 \%$ of participants had a comorbid psychiatric disorder, with $43 \%$ having an anxiety disorder, and $41 \%$ having a mood disorder [11]. Psychiatric disorders are similarly associated with an increased risk of developing OUD in individuals who are prescribed opioids for pain (OR 1.46, 95\% CI 1.12, 1.91) [13].

The importance of understanding a patient's psychiatric comorbidities in the context of their OUD should not be understated. When assessing patients, it may be challenging to determine whether their symptoms are due to a psychiatric comorbidity, or secondary to substance use [14]. These comorbidities also must be considered in the context of treatment. For patients with OUD, psychiatric comorbidities have been associated with worse clinical outcomes. A study examining retention in buprenorphine treatment found the presence of any comorbid mental illness to have a hazard ratio of
1.05 (95\% CI 1.01, 1.10) for treatment discontinuation at three years [15] while inpatient mental health treatment was associated with a hazard ratio of 1.2 (95\% CI 1.19, 1.30) for treatment discontinuation [16]. Similarly, psychiatric comorbidity conferred higher hazard for attrition from methadone treatment (hazard ratio 1.20, 95\% CI 1.06, 1.35) [15]. In studying the impact of psychological symptoms in OUD treatment outcomes, we have previously found that psychological symptoms were associated with higher percentages of opioid-positive drug screens $(B=0.02,95 \%$ CI $0.01-0.02=3)$ and non-opioidpositive drug screens $(B=1.92,95 \%$ CI $0.89,2.95)$ [14]. The prevalence of depression and anxiety disorders in opioid-related overdoses reaches rates greater than $30 \%$ [17], and severe depression (OR 2.46, 95\% CI 1.24, 4.89), post-traumatic stress disorder (PTSD) (OR 2.77, 95\% CI 1.37, 5.60), and psychosis (OR 2.39, 95\% CI 1.10, 5.15) are significantly associated with opioid overdoses [18]. In a study looking at factors associated with mortality among heroin users, a history of psychiatric treatment was associated with a hazard ratio of 6.21 (95\% CI 2.52, 15.33) for all-cause mortality [19].

Psychiatric comorbidity is also associated with lower reported quality of life for patients with OUD [20]. Epidemiological data on patient outcomes, in addition to other clinical and systems-level research, have reinforced the need for concurrent disorders treatment that integrates treatment for psychiatric disorders and addictions $[10,21]$.

Most commonly in substance use treatment programs, the record of historical mental health diagnoses is based on patient self-report [22]. The utility of self-reported psychiatric diagnoses within patients with SUDs was examined by Neumann and colleagues [22] who found self-reported diagnoses of bipolar disorder and depression were often not confirmed by current clinical measures, and many participants had undiagnosed psychiatric disorders. In light of these findings and a clinical reliance on patient self-report, the objective of this study was to examine the sensitivity and specificity of patient-reported psychiatric diagnoses in a cohort of patients receiving outpatient pharmacological treatment for OUD.

\section{Methods}

Data were collected in the GENetics of Opioid Addiction (GENOA) prospective cohort study conducted in Ontario, Canada. The objective of the GENOA study was to examine biological and social factors affecting treatment outcome and course for patients with OUD [23]. Participants were recruited from 20 outpatient opioid agonist treatment clinics between 2011 and 2017. All clinics were run by the same management teams and followed the same treatment protocols. Inclusion criteria required 
participants to be a minimum of 18 years of age, to meet diagnostic criteria for OUD as per DSM-IV criteria, [24] and to be enrolled in opioid agonist therapy (primarily methadone). At the time that this study was conducted, buprenorphine-naloxone was not covered through the Ontario provincial drug plan, thus greatly limiting its access, and making methadone the primary opioid substitution treatment available. No restrictions were placed on participants' duration in treatment; therefore, individuals were enrolled in treatment for different lengths of time at the time of study entry. This study was conducted in accordance with the Declaration of Helsinki Ethical Principles for Medical Research Involving $\mathrm{Hu}$ man Subjects. Ethics approval was obtained from the Hamilton Integrated Research Ethics Board (project ID 11-056) and all participants provided informed consent. We report the present study following the Strengthening the Reporting of Observational Studies in Epidemiology (STROBE) guidelines [25].

At study intake, all participants completed extensive baseline assessments performed by trained interviewers, including assessment of sociodemographic and clinical information (including opioid agonist treatment history). All participants were asked to self-report their medical history, with the open-ended question: "Do you have any medical conditions or health problems?" Responses to this question were recorded in an open-ended text field and later summarized into diagnostic categories by two study authors (KL and EX) in duplicate for consistency and accuracy.

Self-reported psychiatric diagnoses were compared against a diagnostic interview using the Mini Neuropsychiatric Interview (MINI) version 6.0 [26] a standardized and structured tool widely used in research and clinical settings [24]. The MINI has been validated against the Structured Clinical Interview for Diagnostic and Statistical Manual of Mental Disorders (SCID) and the Composite International Diagnostic Interview (CIDI) [26]. In comparison to the SCID, the sensitivity of the MINI was $70 \%$ or greater for 19 out of 22 diagnoses evaluated by the SCID while the specificity was $85 \%$ or higher for all diagnoses. When compared to the CIDI, the MINI had a sensitivity of $70 \%$ or greater for 7 out of 11 diagnoses captured by the CIDI and had a specificity of $70 \%$ or greater for all diagnoses. The MINI had excellent interrater reliability with $70 \%$ of interrater kappa values 0.90 or above. The retest reliability of the MINI was good with $61 \%$ of the retest kappa values being above 0.75 . Additionally, the agreement between diagnoses made by general practitioners using the MINI and diagnoses from psychiatrists was $85 \%$ [26]..

The first 690 participants consecutively recruited into the study were administered the MINI version 6.0 [26]. Use of the MINI was subsequently discontinued due to time burden of administration. The MINI interview encompasses assessments of mood disorders (major depression and bipolar disorder), anxiety disorders (panic disorder, agoraphobia, social anxiety disorder, generalized anxiety disorder), obsessive-compulsive disorder (OCD), PTSD, psychotic disorders (schizophrenia, schizoaffective disorder, schizophreniform disorder, brief psychotic disorder, substance induced psychotic disorder, and psychotic disorder not otherwise specified), and eating disorders (anorexia nervosa and bulimia nervosa). In order to reasonably compare self-reported psychiatric diagnoses with diagnoses identified by structured diagnostic interview, we only considered relevant the diagnoses assessed for in the MINI (for example, attention deficit hyperactivity disorder is not included in the MINI and was therefore excluded from self-report). Antisocial personality disorder is examined in the MINI, however this diagnosis was not self-reported by any of the study participants and was therefore excluded from the present analyses. No other personality disorders are identified using the MINI.

We used descriptive statistics to summarize participant demographic and clinical characteristics, using mean values with standard deviation (SD) or median values with interquartile range (IQR) for continuous variables, and frequencies for categorical variables. Sensitivity and specificity values were calculated for each of the following disorders: any psychiatric disorder, depression, bipolar disorder, anxiety disorder, OCD, PTSD, psychotic disorder, and eating disorder. All analyses were conducted using Stata version 15.1.

\section{Results}

Altogether, 1390 participants were recruited into the GENOA study, and upon exclusion of participants recruited in duplicate $(n=30)$ and participants who did not receive the MINI diagnostic interview $(n=677)$, our final study sample consisted of 683 participants (Fig. 1. Study flow diagram). We summarize participant characteristics in Table 1: the mean age was 39.1 years $(\mathrm{SD}=$ $11.4)$ and males composed $58 \%$ of the cohort $(n=395)$.

The unemployment rate was $66 \%(n=450)$. The reported mean age at first opioid use was 25.5 years $(\mathrm{SD}=$ 8.8 ) and the median years spent in treatment was $3(\mathrm{Q} 1$, $\mathrm{Q} 3=1,6)$. The mean methadone dose for the participants was $77 \mathrm{mg} /$ day $(\mathrm{SD}=46.5)$. Three participants in this study sample were prescribed buprenorphinenaloxone $(0.4 \%)$ at the time of recruitment. At study entry, the percentage of participants that reported being abstinent from opioids was $49 \%(n=335)$. The median percentage of opioid-positive drug screens in participants who reported being non-abstinent at the beginning of the study was $25 \%(\mathrm{Q} 1, \mathrm{Q} 3=10,58.2)$. 


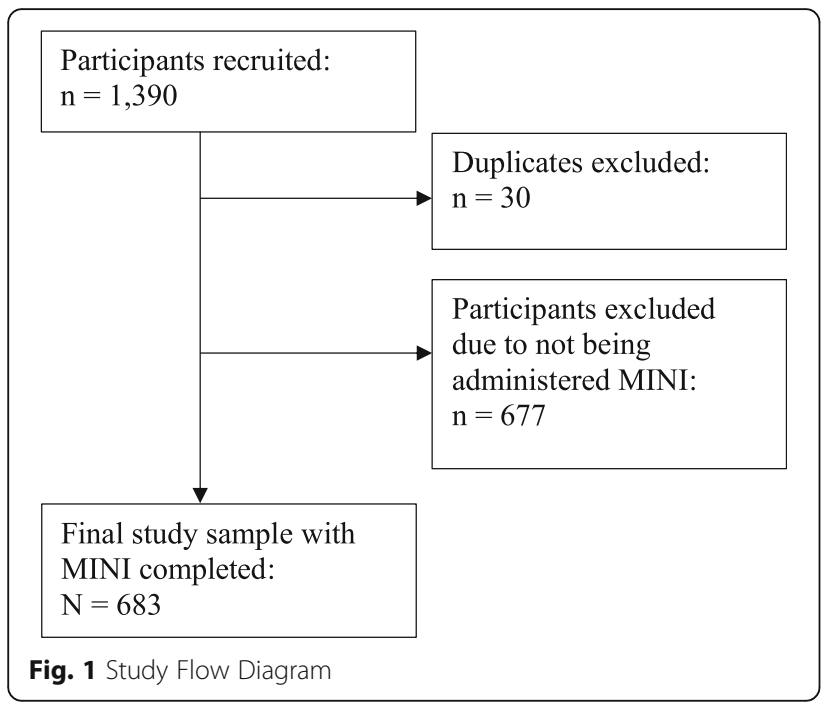

Amongst all study participants, 104 (15.2\%) reported a diagnosis of depression, 15 (2.2\%) reported a diagnosis of bipolar disorder, 102 (14.9\%) reported a diagnosis of an anxiety disorder, and 27 (4.0\%) reported a diagnosis of PTSD. Less frequently reported diagnoses were OCD, psychotic disorders, and eating disorders. In total, 162 (23.7\%) participants self-reported a history of psychiatric comorbidity (Table 2).

The sensitivity and specificity of self-reported psychiatric diagnoses are reported in Table 3.

Of the 162 participants who self-reported any psychiatric comorbidity, 104 met criteria for a comorbidity as per the MINI and 58 did not. Furthermore, 304 participants who reported no psychiatric comorbidity were identified to meet MINI criteria for a psychiatric disorder. The sensitivity and specificity for any selfreported psychiatric diagnoses was $25.5 \%$ (95\% CI 21.3, 30.0 ) and $78.9 \%$ (95\% CI 73.6, 83.6), respectively.
The sensitivity and specificity for self-reported depression was $17.9 \%(95 \%$ CI $12.7,24.1)$ and $85.8 \%(95 \%$ CI 82.4, 88.8), respectively; meanwhile the sensitivity and specificity for self-reported anxiety disorders was $14.8 \%$ (95\% CI 10.5, 20.1) and 85\% (95\% CI 81.4, 88.2), respectively; and the sensitivity and specificity for self-reported PTSD was $4.2 \%$ (95\% CI $0.8,11.7)$ and $96.1 \%$ (95\% CI 94.2, 97.5), respectively. The sensitivity for each of selfreported bipolar disorder, OCD, psychotic disorder, and eating disorders was $0 \%$ (95\% CI for bipolar disorder 0 , 4.0; 95\% CI for OCD 0, 4.7; 95\% CI for psychotic disorder $0,13.7 ; 95 \%$ CI for eating disorder $0,36.9)$. The specificity was $97.5 \%$ for self-reported bipolar disorder (95\% CI 95.9, 98.6), 99.3\% for OCD (95\% CI 98.3, 99.8), 99.4\% for psychotic disorder (95\% CI 98.5, 99.8), and 99.9\% for eating disorders (95\% CI 99.2, 99.996). There were many new diagnoses identified using the MINI including 156 diagnoses of depression, 195 diagnoses of anxiety, 69 diagnoses of PTSD, 90 diagnoses of bipolar disorder, 76 diagnoses of OCD, 25 diagnoses of psychotic disorders, and 8 diagnoses of eating disorders.

\section{Discussion}

Overall, we found that the sensitivity of self-reported psychiatric comorbidity was low, when compared to the MINI, particularly for self-reported depression, anxiety disorder, PTSD, and overall psychiatric diagnoses. In addition, none of the patients with a self-reported history of bipolar disorder, OCD, psychotic disorder, or eating disorder met the criteria for a current diagnosis of each disorder. Many new diagnoses were made in participants who did not report a history of these disorders including but not limited to depression, anxiety, PTSD, and bipolar disorder. Although the specificity of selfreport was higher for certain diagnoses (including anxiety disorder and PTSD), the overall low sensitivity

Table 1 Participant characteristics at study entry $(N=683)$

\begin{tabular}{|c|c|c|c|}
\hline Characteristic & $\begin{array}{l}\text { Total sample } \\
N=683\end{array}$ & $\begin{array}{l}\text { Self-reported psychiatric comorbidity } \\
n=162(23.7 \%)\end{array}$ & $\begin{array}{l}\text { No self-reported psychiatric comorbidity } \\
n=521(76.3 \%)\end{array}$ \\
\hline Age (years); mean (SD) & $39.1(11.4)$ & $37.8(11.6)$ & $39.4(11.3)$ \\
\hline \multicolumn{4}{|l|}{ Sex; n (\%) } \\
\hline Males & 395 (57.8\%) & 94 (58.0\%) & 301 (57.8\%) \\
\hline Females & $288(42.2 \%)$ & 68 (42.0\%) & 220 (42.2\%) \\
\hline Unemployment; $n$ (\%) & $450(65.9 \%)$ & $108(66.7 \%)$ & $342(65.5 \%)$ \\
\hline Age at first opioid use (years); mean (SD) & $25.5(8.8)$ & $25.2(8.7)$ & $25.6(8.8)$ \\
\hline Years in treatment; median $(\mathrm{Q} 1, \mathrm{Q} 3)$ & $3(1,6)$ & $2(1,5)$ & $3(1,7)$ \\
\hline Methadone dose (mg/d); mean (SD) & $77.0(46.5)$ & $71.8(39.8)$ & $78.7(48.3)$ \\
\hline Opioid abstinence at study entry; $n$ (\%) & $335(49.1)$ & $71(43.8)$ & $264(50.7)$ \\
\hline $\begin{array}{l}\text { Percentage }(\%) \text { of opioid-positive drug } \\
\text { screens at study entry if non-abstinent; } \\
\text { median }(\mathrm{Q} 1, \mathrm{Q} 3)\end{array}$ & $25(10,58.2)$ & $29.6(12,64.3)$ & $23.5(9.1,55.6)$ \\
\hline
\end{tabular}

SD Standard Deviation, Q1 Quartile 1, Q3 Quartile 3 
Table 2 Psychiatric comorbidity identified within the study sample $(N=683)$

\begin{tabular}{lll}
\hline Psychiatric comorbidity & Self-reported & MINI-Diagnosed \\
\hline Any psychiatric disorder; $n$ (\%) & $162(23.7 \%)$ & $408(59.7 \%)$ \\
Depression; $n$ (\%) & $104(15.2 \%)$ & $190(27.8 \%)$ \\
Bipolar disorder; $n(\%)$ & $15(2.2 \%)$ & $90(13.2 \%)$ \\
Anxiety disorder; $n$ (\%) & $102(14.9 \%)$ & $229(33.5 \%)$ \\
PTSD; $n$ (\%) & $27(4.0 \%)$ & $72(10.5 \%)$ \\
OCD; $n$ (\%) & $4(0.6 \%)$ & $76(11.1 \%)$ \\
Psychotic disorder; $n(\%)$ & $4(0.6 \%)$ & $25(3.7 \%)$ \\
Eating disorder; $n(\%)$ & $1(0.2 \%)$ & $8(1.2 \%)$ \\
\hline
\end{tabular}

MINI Mini-International Neuropsychiatric Interview, PTSD Post-traumatic Stress Disorder, $O C D$ Obsessive Compulsive Disorder

illustrates that self-report did not accurately capture the patients' present clinical picture. Thus, the utility of selfreported psychiatric comorbidity is in question.

There are several potential explanations for the low sensitivity of self-reported psychiatric disorders. Psychiatric disorders can be episodic in nature, with recurrent symptoms that cycle through relapsing-remitting courses. Participants who reported a historical diagnosis may no longer be experiencing symptoms and no longer meet the criteria for that disorder at the time of assessment. Similarly, participants for whom new psychiatric diagnoses were made may not have previously met criteria for the respective diagnoses. In addition, psychiatric disorders are likely under-diagnosed in the OUD population as evidenced by the under-diagnosis of concurrent disorders as a whole [27]. There may be poor health literacy within the OUD population which can negatively impact an individual's ability to recognize symptoms of a mental disorder and then communicate these issues to a healthcare provider, contributing to underdiagnosis [28]. Furthermore, treatment for mental health and substance abuse disorders continues to be offered separately, despite the evidence supporting concurrent treatment, and between navigating both systems diagnoses may be missed.

Table 3 Sensitivity and specificity of self-reported psychiatric disorders $(N=683)$

\begin{tabular}{lll}
\hline & Sensitivity $\mathbf{( 9 5 \% ~ C l )}$ & Specificity $\mathbf{( 9 5 \% ~ C l )}$ \\
\hline Any psychiatric disorder & $25.5 \%(21.3,30.0)$ & $78.9 \%(73.6,83.6)$ \\
Any depressive disorder & $17.9 \%(12.7,24.1)$ & $85.8 \%(82.4,88.8)$ \\
Bipolar disorder & $0 \%(0,4.0)$ & $97.5 \%(95.9,98.6)$ \\
Any anxiety disorder & $14.8 \%(10.5,20.1)$ & $85 \%(81,4,88.2)$ \\
PTSD & $4.2 \%(0.8,11.7)$ & $96.1 \%(94.2,97.5)$ \\
OCD & $0 \%(0,4.7)$ & $99.3 \%(98.3,99.8)$ \\
Psychotic disorder & $0 \%(0,13.7)$ & $99.4 \%(98.5,99.8)$ \\
Any eating disorder & $0 \%(0,36.9)$ & $99.9 \%(99.2,99.996)$ \\
\hline
\end{tabular}

Cl Confidence Interval, PTSD Post-traumatic Stress Disorder, OCD Obsessive Compulsive Disorder
Most studies examining the sensitivity and specificity of self-reported psychiatric diagnoses do not include participants with SUDs. For instance, studies have found moderate to high agreement between self-reported and clinically-diagnosed depression in groups of university graduates and adults being followed for osteoporosis [29, 30]. Conversely, a self-report tool used to screen members of the United States Armed Forces for deployment was found to have low validity in identifying individuals with diagnosed mental health disorders [31]. Much of the related literature in the patients with SUDs examines self-reported substance use, rather than psychiatric comorbidity [32]. Neumann et al. compared self-reported historical psychiatric diagnoses with current clinical diagnoses assessed using the MINI screen, MINI Plus 500 structured interview, and Personality Diagnostics Questionnaire-4th edition. They found that self-reported diagnoses such as bipolar disorder and depression were often not verified by current clinical measures [22]. In addition, many patients had undiagnosed personality disorders, depression, and anxiety disorders [22]. To our knowledge, no other studies have examined the sensitivity and specificity of self-reported psychiatric diagnoses in SUD or OUD populations.

As discussed, individuals with OUD have higher rates of co-occurring psychiatric comorbidities compared to the general population, with rates reaching greater than $40 \%$ across their lifetime, [12] and these comorbidities are associated with increased psychiatric distress, rates of SUD, and poorer response to substance use treatment $[10,11$, 33]. Despite this, the evidence surrounding the efficacy of psychiatric treatment in these individuals remains equivocal. Literature on pharmacotherapy (mostly consisting of placebo-controlled medication trials) has shown mixed results, with many studies showing no difference from placebo in reducing psychiatric symptoms or substance use [33]. With respect to psychosocial interventions, a Cochrane review found that patients receiving psychosocial interventions in addition to OAT did not show improved outcomes compared to individuals receiving OAT alone; whereas another systematic review did support their efficacy to improve clinical outcomes [34]. There is emerging evidence that some of these inconsistent findings may be indicative of other variables impacting patient responses to psychiatric treatment, including ongoing substance use and complex psychosocial such as housing and employment $[9,33,35]$.

The varied, interlinked, and complex needs of individuals with OUD (and other SUDs) have led to a movement towards concurrent treatment $[10,21]$. While the evidence for how psychiatric treatment impacts outcomes for patients with SUDs is conflicting, there have been encouraging results from literature evaluating integrated care for both psychiatric and SUDs. In patients 
receiving both OAT and psychiatric care, those who received on-site and integrated care of both had improved psychiatric outcomes compared to those who received their psychiatric care off-site and non-integrated with their substance use treatment; however no difference was found in outcomes related to substance use [36]. This remains an area requiring further research to elucidate how to best support these clinically complex patients and improve their outcomes.

For patients experiencing SUDs, clinicians also face challenges differentiating between psychiatric disorders and substance-induced disorders as both intoxication and withdrawal from opioids can mimic the symptomatology seen in mood, anxiety, and psychotic disorders $[12,37,38]$. The difficulty in delineating between psychological symptoms as a result of a primary psychiatric disorder versus secondary to SUD (including OUD) makes diagnosing mental illness in this population a complex challenge [12,37]. In fact, due to the overlap in symptoms between symptom intoxication/withdrawal and affective disorders, Quello et al. advise that definitive assessments of psychiatric diagnoses should be postponed until individuals have achieved a reasonable period of abstinence, although the length of this period varies [39]. Instead they suggest using screening methods to identify possible mood disorders, with associated follow-up after abstinence for definitive diagnosis [39]. Furthermore, individuals may not recall, choose not to disclose, or disagree with a previous psychiatric diagnosis; in addition to selfdiagnosing themselves incorrectly. This could occur for various reasons relating to the social stigma of substance use, the impacts of chronic substance use or untreated psychiatric disorders on memory, lack of access to healthcare for appropriate psychiatric assessment, among others.

Our results highlight the need for improved methods to ascertain comorbid psychiatric disorders in the OUD population during treatment. While the specificities of many psychiatric disorders were high, the sensitivities remained poor. That several patients in this study received new diagnoses additionally highlights the issue of under-diagnosis of psychiatric comorbidities in this population, and the need for better ways to identify them [27]. Potential alternatives to patient self-report of psychiatric diagnoses may include structured interviews or questionnaires. In the setting of an addiction treatment clinic, time and resource limitations, and clinical expertise influence the ability to conduct diagnostic interviews and comprehensively assess psychiatric symptomatology. The GENOA study, from which our data were obtained, initially used the MINI structured diagnostic interview, which takes approximately $20 \mathrm{~min}$ to administer and use of this tool was later discontinued due to the time burden of administration on participants who did not like the additional $20 \mathrm{~min}$ it took to complete the interview. However, the MINI is one of the quickest diagnostic tools used to assess for psychiatric disorder, and other standardized assessments such as the CIDI [40] or the SCID [41] require more time to administer compared to the MINI [42].

Since diagnostic tools require significant time resources, an alternative option would be to administer screening tools that take less time to complete in order to identify the patients who may benefit from a more detailed diagnostic interview. This would be particularly pertinent in relation to the low sensitivities of our results as a way to provide diagnostic clarification when patients self-report psychiatric concerns. Examples of screening tools which require less than $10 \mathrm{~min}$ to complete include the Brief Symptom Inventory, [43] Modified MINI screen, [44] and the General Health Questionnaire [45]. The Dual Diagnosis Screening Instrument [46] and Mental Health Screening Form-III [47] are both screening tools that have been developed specifically for the detection of psychiatric comorbidity in patients with SUDs. Another potential solution would be to have a psychiatrist on-site who would be able to administer assessments for psychiatric disorders, which has also been shown to increase patient engagement with psychiatric services [48].

A limitation of this study was the way self-reported psychiatric disorders were elicited, with an open-ended question inquiring about any medical or health problems. This question may have been understood as asking for solely non-psychiatric medical problems or only for current issues that the patient is experiencing, not past diagnoses that are now in remittance. A question that explicitly asks around a history of mental health disorders both past and present would be less susceptible to misinterpretation. Additionally, the MINI does not assess for personality disorders, which are heavily implicated in SUD, [49] or attention deficit hyperactivity disorder. Thus, analysis regarding the sensitivity and specificity of self-report for these disorders is unavailable. Importantly, there is an under-reporting and under-recognition of psychiatric comorbidities in the context of OUD despite the demonstrated impact of such comorbidity on treatment outcomes. Health care providers need to be aware of the prevalence of such disorders in this population and policies regarding systematic screening of patients attending treatment for SUDs for comorbid psychiatric conditions are needed. The choice of a screening tool or clinical assessment that is feasible to use must balance accuracy, effort, and scalability. It is also possible that for some patients, poor mental health literacy in the SUD population likely further exacerbates the issue of self-recognition of 
psychiatric symptoms and subsequently seeking help with diagnosis.

\section{Conclusion}

The sensitivity and specificity of self-reported psychiatric comorbidity in patients with OUD was found to be low when compared to standardized, structured, diagnostic interviews. The remitting-relapsing nature of psychiatric disorders, under-diagnosis, the difficulty in differentiating independent psychiatric disorders from substanceinduced disorders, and patient factors likely contributed to these findings. These findings highlight that other methods should implemented in order to identify comorbid psychiatric disorders in patients with OUD.

\begin{abstract}
Abbreviations
OUD: Opioid use disorder; MINI: Mini International Neuropsychiatric Interview; SUD: Substance use disorder; GENOA: GENetics of Opioid Addiction; SCID: Structured Clinical Interview for Diagnostic and Statistical Manual of Mental Disorders; CIDI: Composite International Diagnostic Interview; OCD: Obsessive compulsive disorder; PTSD: Post-traumatic stress disorder
\end{abstract}

\section{Supplementary Information}

The online version contains supplementary material available at https://doi. org/10.1186/s12888-021-03489-4.

Additional file 1: Appendix A. Paired contingency tables comparing results of self-reported diagnosis to MINI diagnosis.

\section{Acknowledgements}

We thank the GENOA study participants for their participation and contributions to this study.

\section{Authors' contributions}

$T R$, and ZS are responsible for the study concept and design. $\mathrm{KL}$ and $\mathrm{EX}$ cleaned and prepared the data for analysis and TR conducted statistical analyses. $K L$ and $E X$ wrote the first draft of the manuscript, and TR, AW, LT, and ZS contributed to writing and critically revising the final manuscript. All authors reviewed and approved the final manuscript.

\section{Funding}

This study was supported by research grants from the Canadian Institutes of Health Research (ZS, grant number PJT-153429) and the Hamilton Academic Health Sciences Organization (ZS, grant number HAH-16-04). The funding bodies have no role in the study design, data analysis, interpretation, or publication of results.

\section{Availability of data and materials}

The data that support the findings of this study are available from the corresponding author, $\mathrm{ZS}$, upon reasonable request.

\section{Declarations}

\section{Ethics approval and consent to participate}

This study was conducted in accordance with the Declaration of Helsink Ethical Principles for Medical Research Involving Human Subjects. Ethics approval was obtained from the Hamilton Integrated Research Ethics Board (project ID 11-056). All participants were provided informed consent.

\section{Consent for publication}

Not applicable.

\section{Competing interests}

The authors report no conflict of interest.

\section{Author details}

Michael G. DeGroote School of Medicine, McMaster University, Hamilton, Ontario, Canada. ${ }^{2}$ Department of Psychiatry and Behavioral Neurosciences, McMaster University, Hamilton, Ontario, Canada. ${ }^{3}$ Department of Health Research Methods, Evidence, and Impact, McMaster University, Hamilton, Ontario, Canada. ${ }^{4}$ Biostatistics Unit, Research Institute at St Joseph's Healthcare, Hamilton, Ontario, Canada. ${ }^{5}$ Departments of Pediatrics/ Anesthesia, McMaster University, Hamilton, Ontario, Canada.

Received: 30 June 2021 Accepted: 17 September 2021

Published online: 21 October 2021

\section{References}

1. Degenhardt L, Grebely J, Stone J, Hickman M, Vickerman P, Marshall BDL, et al. Global patterns of opioid use and dependence: harms to populations, interventions, and future action. Lancet. 2019;394(10208):1560-79. https:// doi.org/10.1016/S0140-6736(19)32229-9.

2. Demir B, Altindag A. Naltrexone implant use in opioid use disorder; an example from Turkey. Heroin Addict Relat Clin Problems. 2021;23(4):43-50.

3. Demir B, Şahin ŞK, Elboga G, Altindag A, Unal A. Substance use characteristics, treatment completion rates and related factors of patients in Gaziantep AMATEM in 2019; a retrospective study. J Subst Abus. 2021:1-7. https://doi.org/10.1080/14659891.2021.1912202.

4. Demir B, Altindag A. Is there a relationship between heroin dependence and Anemia? Addictive Disorders \& Their Treatment. 2021.

5. Overdoses SACotEoO. National report: apparent opioid-related deaths in Canada (January 2016 to march 2020). Ottawa: Public Health Agency of Canada; 2020

6. Overdoses SACotEoO. National report: opioid-related poisoning hospitalizations (January 2016 to march 2020). Ottawa: Public Health Agency of Canada; 2020.

7. Ahmad F, Rossen L, Sutton P. Provisional drug overdose death counts. National Center for Health Statistics; 2020.

8. Centers for Disease Control and Prevention NCfHS, National Vital Statistics System „, File M. Number and age-adjusted rates of drug-poisoning deaths involving opioid analgesics and heroin: United States, 2000-2014. Atlanta: Centers for Disease Control and Prevention; 2015.

9. Wen LS, Sadeghi NB. The opioid crisis and the 2020 US election: crossroads for a national epidemic. Lancet. 2020;396(10259):1316-8. https://doi.org/10.1 016/S0140-6736(20)32113-9.

10. Rush B, Urbanoski K, Bassani D, Castel S, Wild TC, Strike C, et al. Prevalence of co-occurring substance use and other mental disorders in the Canadian population. Can J Psychiatr. 2008;53(12):800-9. https://doi.org/10.1177/ 070674370805301206

11. Regier DA, Farmer ME, Rae DS, Locke BZ, Keith SJ, Judd LL, et al. Comorbidity of mental disorders with alcohol and other drug abuse. Results from the epidemiologic catchment area (ECA) study. Jama. 1990;264(19): 2511-8. https://doi.org/10.1001/jama.1990.03450190043026.

12. Strain EC. Assessment and treatment of comorbid psychiatric disorders in opioid-dependent patients. Clin J Pain. 2002;18(4 Suppl):S14-27. https://doi. org/10.1097/00002508-200207001-00003.

13. Edlund MJ, Steffick D, Hudson T, Harris KM, Sullivan M. Risk factors for clinically recognized opioid abuse and dependence among veterans using opioids for chronic non-cancer pain. Pain. 2007;129(3):355-62. https://doi. org/10.1016/j.pain.2007.02.014.

14. Rosic T, Worster A, Thabane L, Marsh DC, Samaan Z. Exploring psychological symptoms and associated factors in patients receiving medication-assisted treatment for opioid-use disorder. BJPsych Open. 2020;6(1):e8. https://doi. org/10.1192/bjo.2019.99

15. Manhapra A, Agbese E, Leslie DL, Rosenheck RA. Three-year retention in buprenorphine treatment for opioid use disorder among privately insured adults. Psychiatr Serv. 2018;69(7):768-76. https://doi.org/10.1176/appi.ps.201 700363.

16. Mancino M, Curran G, Han X, Allee E, Humphreys K, Booth BM. Predictors of attrition from a national sample of methadone maintenance patients. Am J Drug Alcohol Abuse. 2010;36(3):155-60. https://doi.org/10.3109/00952991 003736389

17. Smolina K, Crabtree A, Chong M, Zhao B, Park M, Mill C, et al. Patterns and history of prescription drug use among opioid-related drug overdose cases in British Columbia, Canada, 2015-2016. Drug Alcohol Depend. 2019;194 151-8. https://doi.org/10.1016/j.drugalcdep.2018.09.019. 
18. Fendrich M, Becker J, Hernandez-Meier J. Psychiatric symptoms and recent overdose among people who use heroin or other opioids: results from a secondary analysis of an intervention study. Addict Behav Rep. 2019;10: 100212. https://doi.org/10.1016/j.abrep.2019.100212.

19. Huang $\mathrm{CL}$, Lee $\mathrm{CW}$. Factors associated with mortality among heroin users after seeking treatment with methadone: a population-based cohort study in Taiwan. J Subst Abus Treat. 2013;44(3):295-300. https://doi.org/10.1016/j. jsat.2012.08.003.

20. Carpentier PJ, Krabbe PF, van Gogh MT, Knapen LJ, Buitelaar JK, de Jong CA. Psychiatric comorbidity reduces quality of life in chronic methadone maintained patients. Am J Addict. 2009;18(6):470-80. https://doi.org/10.31 09/10550490903205652.

21. Davidson $L$, White $W$. The concept of recovery as an organizing principle for integrating mental health and addiction services. J Behav Health Serv Res. 2007:34(2):109-20. https://doi.org/10.1007/s11414-007-9053-7.

22. Neumann AM, Giambrone AK, Yanes PK, Jaanimägi U, Wisniewski AM, Valtin $L L$, et al. Validity of self-reported psychiatric diagnoses among patients seeking treatment for a substance use disorder. Ment Health Subst Use. 2013;6(2):111-23. https://doi.org/10.1080/17523281.2012.693129.

23. Samaan Z, Bawor M, Dennis BB, Plater C, Varenbut M, Daiter J, et al. Genetic influence on methadone treatment outcomes in patients undergoing methadone maintenance treatment for opioid addiction: a pilot study. Neuropsychiatr Dis Treat. 2014;10:1503-8. https://doi.org/10.2147/NDT. S66234.

24. American Psychiatric Association. Diagnostic and Statistical Manual of Mental Disorders (4th edn, revised) (DSM-IV-TR). American Psychiatric Publishing; 2000.

25. von Elm E, Altman DG, Egger M, Pocock SJ, Gøtzsche PC, Vandenbroucke JP. The strengthening the reporting of observational studies in epidemiology (STROBE) statement: guidelines for reporting observational studies. Int I Surg. 2014;12(12):1495-9. https://doi.org/10.1016/j.jjsu.2014.07.013.

26. Sheehan DV, Lecrubier $Y$, Sheehan $\mathrm{KH}$, Amorim $\mathrm{P}$, Janavs J, Weiller $E$, et al. The Mini-International Neuropsychiatric Interview (M.I.N.I.): the development and validation of a structured diagnostic psychiatric interview for DSM-IV and ICD-10. J Clin Psychiatry. 1998;59 Suppl 20:22-33;quiz 4-57.

27. Hakobyan S, Vazirian S, Lee-Cheong S, Krausz M, Honer WG, Schutz CG. Concurrent Disorder Management Guidelines. Systematic Review. J Clin Med. 2020;9(8):2406.

28. Rogers AH, Bakhshaie J, Orr MF, Ditre JW, Zvolensky MJ. Health literacy, opioid misuse, and pain experience among adults with chronic pain. Pain Med. 2020;21(4):670-6. https://doi.org/10.1093/pm/pnz062.

29. Sanchez-Villegas A, Schlatter J, Ortuno F, Lahortiga F, Pla J, Benito S, et al. Validity of a self-reported diagnosis of depression among participants in a cohort study using the structured clinical interview for DSM-IV (SCID-I). BMC Psychiatry. 2008;8(1):43. https://doi.org/10.1186/1471-244X-8-43.

30. Stuart AL, Pasco JA, Jacka FN, Brennan SL, Berk M, Williams LJ. Comparison of self-report and structured clinical interview in the identification of depression. Compr Psychiatry. 2014;55(4):866-9. https://doi.org/10.1016/j. comppsych.2013.12.019.

31. Nevin RL. Low validity of self-report in identifying recent mental health diagnosis among U.S. service members completing Pre-Deployment Health Assessment (PreDHA) and deployed to Afghanistan, 2007: a retrospective cohort study. BMC Public Health. 2009;9:376.

32. Jackson CT, Covell NH, Frisman LK, Essock SM. Validity of self-reported drug use among people with co-occurring mental health and substance use disorders. J Dual Diag. 2005;1(1):49-63. https://doi.org/10.1300/J374v01n01_05.

33. Kidorf M, King VL, Peirce J, Gandotra N, Ghazarian S, Brooner RK. Substance use and response to psychiatric treatment in methadone-treated outpatients with comorbid psychiatric disorder. J Subst Abus Treat. 2015;51: 64-9. https://doi.org/10.1016/j.jsat.2014.10.012.

34. Dugosh K, Abraham A, Seymour B, McLoyd K, Chalk M, Festinger D. A systematic review on the use of psychosocial interventions in conjunction with medications for the treatment of opioid addiction. J Addict Med. 2016; 10(2):93-103. https://doi.org/10.1097/ADM.0000000000000193.

35. Carpenter KM, Brooks AC, Vosburg SK, Nunes EV. The effect of sertraline and environmental context on treating depression and illicit substance use among methadone maintained opiate dependent patients: a controlled clinical trial. Drug Alcohol Depend. 2004;74(2):123-34. https://doi.org/10.101 6/j.drugalcdep.2003.11.015.

36. Brooner RK, Kidorf MS, King VL, Peirce J, Neufeld K, Stoller K, et al. Managing psychiatric comorbidity within versus outside of methadone treatment settings: a randomized and controlled evaluation. Addiction. 2013;108(11): 1942-51. https://doi.org/10.1111/add.12269.

37. Schuckit MA. Comorbidity between substance use disorders and psychiatric conditions. (0965-2140 (Print)).

38. Kadden RM, Kranzler HR, Rounsaville BJ. Validity of the distinction between "substance-induced" and "independent" Depressio and anxiety disorders. Am J Addict. 1995;4(2):107-17. https://doi.org/10.3109/10550499508997432.

39. Quello SB, Brady KT, Sonne SC. Mood disorders and substance use disorder: a complex comorbidity. Science \& practice perspectives. 2005;3(1):13-21. https://doi.org/10.1151/spp053113.

40. Robins LN, Wing J, Wittchen HU, Helzer JE, Babor TF, Burke J, et al. The composite international diagnostic interview. An epidemiologic instrument suitable for use in conjunction with different diagnostic systems and in different cultures. Arch Gen Psychiatry. 1988;45(12):1069-77. https://doi. org/10.1001/archpsyc.1988.01800360017003.

41. Spitzer RL, Williams JB, Gibbon M, First MB. The structured clinical interview for DSM-III-R (SCID). I: history, rationale, and description. Arch Gen Psychiatry. 1992;49(8):624-9. https://doi.org/10.1001/archpsyc.1992.0182 0080032005.

42. Levis B, McMillan D, Sun Y, He C, Rice DB, Krishnan A, et al. Comparison of major depression diagnostic classification probability using the SCID, CIDI, and MINI diagnostic interviews among women in pregnancy or postpartum: an individual participant data meta-analysis. Int J Methods Psychiatr Res. 2019;28(4):e1803. https://doi.org/10.1002/mpr.1803.

43. Derogatis $L R$, Melisaratos $N$. The brief symptom inventory: an introductory report. Psychol Med. 1983;13(3):595-605. https://doi.org/10.1017/S0033291 700048017.

44. Alexander MJ, Haugland G, Lin SP, Bertollo DN, McCorry FA. Mental health screening in addiction, corrections and social service settings: validating the MMS. Int J Ment Heal Addict. 2008;6(1):105-19. https://doi.org/10.1007/s114 69-007-9100-X.

45. Goldberg DP, Williams $P$, University of $L$, Institute of $P$. A user's guide to the General Health Questionnaire: NFER-NELSON; 1991.

46. Mestre-Pintó Jl, Domingo-Salvany A, Martín-Santos R, Torrens M. Dual diagnosis screening interview to identify psychiatric comorbidity in substance users: development and validation of a brief instrument. Eur Addict Res. 2014;20(1):41-8. https://doi.org/10.1159/000351519.

47. Carroll JFX, McGinley JJ. A screening form for identifying mental health problems in alcohol/other drug dependent persons. Alcohol Treat Q. 2001; 19(4):33-47. https://doi.org/10.1300/J020v19n04_02.

48. Friedmann PD, D'Aunno TA, Jin L, Alexander JA. Medical and psychosocial services in drug abuse treatment: do stronger linkages promote client utilization? Health Serv Res. 2000;35(2):443-65.

49. Rounsaville BJ, Kranzler HR, Ball S, Tennen H, Poling J, Triffleman E. Personality disorders in substance abusers: relation to substance use. J Nerv Ment Dis. 1998;186(2):87-95. https://doi.org/10.1097/00005053-19980200000004.

\section{Publisher's Note}

Springer Nature remains neutral with regard to jurisdictional claims in published maps and institutional affiliations.

\section{Ready to submit your research? Choose BMC and benefit from:}

- fast, convenient online submission

- thorough peer review by experienced researchers in your field

- rapid publication on acceptance

- support for research data, including large and complex data types

- gold Open Access which fosters wider collaboration and increased citations

- maximum visibility for your research: over $100 \mathrm{M}$ website views per year

At BMC, research is always in progress.

Learn more biomedcentral.com/submissions 